\title{
Pars Plana Vitrectomy Combined with Internal Limiting Membrane Peeling to Treat Persistent Macular Edema after Anti-Vascular Endothelial Growth Factor Treatment in Cases of Ischemic Central Retinal Vein Occlusion
}

\author{
Yukari Shirakata $^{\mathrm{a}}$ Tomoyoshi Fujita $^{\mathrm{a}}$ Yuki Nakano $^{\mathrm{a}}$ Fumio Shiraga $^{\mathrm{b}}$ \\ Akitaka Tsujikawa \\ ${ }^{a}$ Department of Ophthalmology, Kagawa University Faculty of Medicine, Takamatsu, and \\ ${ }^{b}$ Department of Ophthalmology, Okayama University, Okayama, Japan
}

\section{Key Words}

Anti-vascular endothelial growth factor $\cdot$ Central retinal vein occlusion $\cdot$ Internal limiting membrane peeling $\cdot$ Macular edema $\cdot$ Pars plana vitrectomy

\begin{abstract}
Objective: To evaluate the efficacy of pars plana vitrectomy (PPV) combined with internal limiting membrane (ILM) peeling in cases of ischemic central retinal vein occlusion (CRVO) where macular edema (ME) persisted after anti-vascular endothelial growth factor (antiVEGF) treatment. Methods: Fifteen eyes with ischemic CRVO-related ME were included in the study. Nine were treated with panretinal photocoagulation after initial examination. AntiVEGF agents were injected intravitreally. Persistent ME was treated with PPV combined with ILM peeling. During surgery, laser photocoagulation was further applied to the non-perfused area. Results: Mean retinal thickness gradually decreased after surgery $(p=0.024$ at 6 months), although visual acuity did not improve significantly during the follow-up period (14.7 \pm 11.6 months). Neovascular glaucoma subsequently developed in three cases and a trabeculectomy was performed in one case. Conclusion: In eyes with ischemic CRVO, PPV combined with ILM peeling contributed to a reduction in persistent ME. However, there was no significant improvement in visual acuity.


Case Reports in

Ophthalmology
Case Rep Ophthalmol 2016;7:1-8

\begin{tabular}{|l|l}
\hline DOI: $10.1159 / 000443322$ & C 2016 The Author(s). Published by S. Karger AG, Basel
\end{tabular} www.karger.com/cop

Shirakata et al.: PPV Combined with ILM Peeling to Treat Persistent Macular Edema after Anti-VEGF Treatment in Cases of Ischemic Central Retinal Vein Occlusion

\section{Introduction}

Central retinal vein occlusion (CRVO) is often accompanied by various vision-threatening complications, including macular edema (ME), neovascular glaucoma or macular ischemia [1]. Despite various treatments, ME resulting from CRVO often results in severely impaired visual function. Vascular endothelial growth factor (VEGF) has been reported to play an important role in the pathogenesis of CRVO-related ME [2, 3]. Furthermore, an increasing number of investigators have reported the efficacy of anti-VEGF treatment in this condition, and this treatment has since become a standard [4, 5].

Anti-VEGF agents rapidly increase the absorption of ME. However, in most cases, eyes require repeated injections if the effects are to be maintained. In the HORIZON trial [6], for example, the mean number of injections of ranibizumab was 3.5-3.8 in the second year of treatment, yet improvements in visual acuity (VA) had actually decreased in number by the end of that year. More recently, Hayreh [1] observed 697 CRVO-affected eyes and reported that final VA was worse in $85 \%$ of ischemic CRVO cases and $17 \%$ of non-ischemic CRVO cases. Thus, ischemic CRVO cases are associated with a poorer visual prognosis.

Previously, several investigators have reported the anatomical and functional efficacy of pars plana vitrectomy (PPV) combined with internal limiting membrane (ILM) peeling in CRVO-associated ME [7-10]. However, some also reported that a reduction in ME is not accompanied by visual improvement [11-13]. In cases of ME that have not been improved by repeated anti-VEGF treatment, surgical intervention may be a viable treatment option. Despite this, limited information is currently available regarding the feasibility of surgery after anti-VEGF treatment in cases of ischemic CRVO-related ME [14].

In this study, we retrospectively examined the efficacy of PPV combined with ILM peeling in cases of ischemic CRVO where ME had persisted in spite of anti-VEGF treatments.

\section{Patients and Methods}

For this retrospective study, we reviewed the medical records of 15 ischemic CRVO patients who had undergone unilateral PPV combined with ILM peeling as a treatment for ME that had persisted after anti-VEGF treatment. There were 7 women and 8 men among the patients and a total of 15 eyes were examined (table 1). The treatments took place at Kagawa University Hospital between May 2010 and May 2014. Patients were offered the surgery if they had suffered visual loss as a result of persistent ME. They were excluded from the study if they showed proliferative diabetic retinopathy, branch retinal vein occlusion, dense cataract or a short follow-up period of $<6$ months after the surgery. Cases in whom there had been previous treatments for ME or CRVO were also excluded from the study.

CRVO and ME were diagnosed by fundus examination and confirmed by fluorescein angiography and optical coherence tomography (OCT). After the medical history had been obtained, each patient underwent a complete ophthalmological examination, including bestcorrected VA measurement with a Landolt chart, slit-lamp biomicroscopy, indirect fundus ophthalmoscopy and OCT examination. Digital fundus photographs and fluorescein angiography were obtained from each patient, after pupil dilatation, using a digital fundus camera (TRC-50LX; Topcon, Tokyo, Japan) or an Optos P200 Scanning Laser Ophthalmoscope (Optos North America, Marlborough, Mass., USA).

Ischemic CRVO was confirmed at the initial visit by detecting a non-perfused region of $>10$ disc areas using fluorescein angiography. Repeat fluorescein angiography was performed if necessary. To evaluate the ME status, an OCT examination was performed (Cirrus; 
Carl Zeiss, Dublin, Calif., USA) at each visit. This included vertical and horizontal crosssectional scans centered on the fovea. Central retinal thickness (CRT) was defined as the average retinal thickness in a circular region of the fovea with a diameter of $1 \mathrm{~mm}$.

Patients who had a visual disturbance due to CRVO-associated ME were initially given an intravitreal injection of $1.25 \mathrm{mg}$ bevacizumab (Avastin; Genentech, South San Francisco, Calif., USA) or 0.5 mg ranibizumab (Lucentis; Novartis, Bülach, Switzerland), which has now been approved in Japan. An initial CRT $>300 \mu \mathrm{m}$ was required for inclusion in the study. Pseudophakic eyes were also included, but eyes that had undergone a prior vitrectomy were excluded. A retreatment was performed when eyes showed recurrence of ME with visual loss and when the patient agreed to the additional treatment.

All eyes in this study underwent a standard 25-gauge three-port PPV for the treatment of recurrent or persistent ME. After core vitrectomy, posterior vitreous detachment was induced if the cortical vitreous was adherent to the retina. The ILM was peeled approximately $3 \times 3$ disc diameters around the fovea with the aid of indocyanine green or brilliant blue $G$ dye. During surgery, laser photocoagulation was performed on the non-perfused area. In 13 phakic eyes, phacoemulsification and intraocular lens implantation were also performed.

Statistical analysis was performed using IBM SPSS Statistics version 21.0 (IBM, Chicago, Ill., USA). Values are presented as mean \pm standard deviation. For statistical analysis, VA measured with a Landolt chart was converted to a logarithm of the minimum angle of resolution ( $\log M A R)$. Analysis of variance of repeated measurements was used to analyze CRT and VA after the initiation of treatment. A $p$ value $<0.05$ was considered statistically significant.

\section{Results}

All eyes showed ME with symptomatic visual disturbance at the initial visit. VA ranged from 0.40 to 2.00 (average $0.88 \pm 0.57$ ) in logMAR.

After comprehensive ophthalmic examinations, nine of the patients were treated with panretinal photocoagulation. All eyes were treated with an intravitreal injection of antiVEGF agents (bevacizumab in 14 eyes and ranibizumab in 1 eye). After this anti-VEGF treatment, a reduction in ME was achieved. Compared with baseline, CRT had decreased significantly after 1 month $(\mathrm{p}=0.021)$. However, VA had not significantly improved over the same time period $(p=0.769)$. In spite of repeated injections, all eyes showed persistent or recurrent ME. The mean number of injections (bevacizumab or ranibizumab) was $2.27 \pm 1.34$ (table 2). Three of the patients were also treated with a sub-Tenon injection of triamcinolone acetonide.

All patients were then treated with PPV and ILM peeling. Table 3 shows the change in CRT and VA after surgery. CRT decreased gradually and this decrease became significantly different from the presurgical value after 6 months $(p=0.024)$. In spite of this reduction in CRT, VA showed no significant improvement throughout the follow-up period $(14.7 \pm 11.6$ months) (fig. 1). Three eyes subsequently developed neovascular glaucoma and one patient underwent trabeculectomy.

\section{Discussion}

Since the Central Vein Occlusion Study Group [15] cast doubt on the efficacy of grid laser photocoagulation for chronic CRVO-related ME, no effective alternative treatment standard 
Shirakata et al.: PPV Combined with ILM Peeling to Treat Persistent Macular Edema after Anti-VEGF Treatment in Cases of Ischemic Central Retinal Vein Occlusion

had been established. The SCORE study [16] then demonstrated the efficacy of intravitreal triamcinolone for ME secondary to perfused CRVO. However, the proportion of patients who had a gain in VA letter score of $\geq 15$ from baseline to month 12 was $<30 \%$ [16]. Currently, anti-VEGF treatment is generally thought to be the best choice for CRVO-associated ME [4]. In the CRUISE study [17], the mean improvement in VA was 12.7 and 14.9 letters with 6 monthly injections of ranibizumab ( 0.3 and $0.5 \mathrm{mg}$, respectively). However, most eyes required repeated injections. In the HORIZON trial [6], the mean number of injections of ranibizumab was $>3$ in the second year after the initiation of the treatment. The RETAIN study [18] concluded that a substantial minority (44\%) of patients with ranibizumab-treated CRVO had edema resolution and a good outcome within 4 years, but most (56\%) still required frequent injections and had reduced visual potential and a guarded prognosis. Taken together, these studies suggest that although anti-VEGF treatment for ME is convenient and has a rapid effect, repeated injections can be a burden for patients.

In this study, PPV with ILM peeling caused a gradual reduction in ME. However, the mechanism by which this reduction was achieved is still uncertain. Vitrectomy may ameliorate retinal ischemia by allowing oxygenated fluid to circulate in the vitreous cavity [19]. Vitreomacular attachment is also suggested to be involved in persistent ME in eyes with CRVO. For example, Kado et al. [20] reported that ME lasted significantly longer in CRVO eyes with vitreomacular attachment than in those without. In addition, the occurrence of posterior vitreous detachment may function primarily to facilitate the absorption of ME associated with CRVO [21]. ILM peeling, on the other hand, may contribute to the process by ensuring the complete absence of traction in the macular area.

Several reports have shown the anatomical and functional efficacy of PPV combined with ILM peeling on ME in eyes with CRVO [7-10]. However, some reported that the reduction of ME was not accompanied by visual improvement [11-13]. In this study, our patients' ME gradually decreased after surgery, yet there was no significant improvement in VA. In a previous analysis of eyes with CRVO, Ota et al. [22] reported that substantial damage to the foveal photoreceptor layer was associated with poor VA prognosis. In their report, the integrity of the foveal photoreceptor layer after resolution of ME was significantly correlated with initial retinal perfusion status and initial VA. All our patients initially had extensive nonperfused retina and initial VA was poor. Therefore, the findings of Ota et al. may explain the anatomical and functional disparity seen in our patients.

The baseline report in the Central Vein Occlusion study found that the median VA of group $\mathrm{N}$ (at least 10 disc areas of retinal non-perfusion) was 20/400 [23]. Furthermore, the $\mathrm{N}$ report of the study showed that approximately one-third of eyes had no change in VA, onethird had improved by at least two lines and one-third had lost two or more lines during a 3-year follow-up [24]. In eyes with ischemic CRVO, visual function is severely impaired and recovery of VA may be limited [1]. Recently, the GALILEO study [25] reported that CRVO eyes with non-perfused retina lost a mean of $8.0 \pm 15.8$ letters at 52 weeks without treatment, but that eyes with subsequent aflibercept treatment gained VA by $17.4 \pm 16.1$ letters. Repeated treatment with anti-VEGF agents may improve visual prognosis in eyes with ischemic CRVO. However, this beneficial effect would be limited for eyes with extremely poor initial VA [26].

Recently, the European VitreoRetinal Society ME study [27] reported the efficacy of different therapies in the treatment of ME associated with CRVO [28]. Taking all 358 CRVO cases together, PPV combined with ILM peeling resulted in a greater improvement in vision than other therapies. However, this report showed no information on the perfusion status of the treated eyes. As discussed above, visual prognosis in eyes with persistent ME of eyes 
Shirakata et al.: PPV Combined with ILM Peeling to Treat Persistent Macular Edema after Anti-VEGF Treatment in Cases of Ischemic Central Retinal Vein Occlusion

associated with ischemic CRVO would be poor, even if treated with PPV combined with ILM peeling.

There were various limitations to this study, namely the small sample size and the retrospective study design. The non-comparative design of this study also rendered it impossible to determine whether surgical intervention improved the visual prognosis. Moreover, since cataract surgery was performed in 13 patients, VA results in our patients may be difficult to interpret. However, the effect of cataract surgery on VA improvement would be small. Nevertheless, postoperative improvement on VA was low after 1 month.

In conclusion, PPV combined with ILM peeling showed anatomical efficacy for ME that persisted after anti-VEGF treatment in eyes with ischemic CRVO. However, there was no significant concomitant improvement in VA. Further studies are necessary to elucidate the optimal treatment for persistent ME after anti-VEGF treatment in eyes with ischemic CRVO.

\section{Acknowledgements}

This study was supported in part by the Japan Society for the Promotion of Science (Tokyo, Japan, Grant-in-Aid for Scientific Research).

\section{Statement of Ethics}

Off-label use of bevacizumab was approved by the Institutional Ethics Committee. This study adhered to the tenets of the Declaration of Helsinki. Written informed consent was obtained from each patient.

\section{Disclosure Statement}

The authors have nothing to disclose.

\section{References}

1 Hayreh SS: Ocular vascular occlusive disorders: natural history of visual outcome. Prog Retin Eye Res 2014;41:1-25.

2 Noma H, Funatsu H, Mimura T, Harino S, Hori S: Aqueous humor levels of vasoactive molecules correlate with vitreous levels and macular edema in central retinal vein occlusion. Eur J Ophthalmol 2010;20:402409.

- Noma H, Funatsu H, Mimura T, Harino S, Hori S: Vitreous levels of interleukin-6 and vascular endothelial growth factor in macular edema with central retinal vein occlusion. Ophthalmology 2009;116:87-93.

4 Huang P, Niu W, Ni Z, Wang R, Sun X: A meta-analysis of anti-vascular endothelial growth factor remedy for macular edema secondary to central retinal vein occlusion. PLoS One 2013;8:e82454.

5 Pacella E, Pacella F, La Torre G, Impallara D, Malarska K, Brillante C, Turchetti P, De Giusti M: Testing the effectiveness of intravitreal ranibizumab during 12 months of follow-up in venous occlusion treatment. Clin Ter 2012;163:e413-e422.

6 Heier JS, Campochiaro PA, Yau L, Li Z, Saroj N, Rubio RG, Lai P: Ranibizumab for macular edema due to retinal vein occlusions: long-term follow-up in the HORIZON trial. Ophthalmology 2012;119:802-809.

-7 Park DH, Kim IT: Long-term effects of vitrectomy and internal limiting membrane peeling for macular edema secondary to central retinal vein occlusion and hemiretinal vein occlusion. Retina 2010;30:117-124.

-8 Raszewska-Steglinska M, Gozdek P, Cisiecki S, Michalewska Z, Michalewski J, Nawrocki J: Pars plana vitrectomy with ILM peeling for macular edema secondary to retinal vein occlusion. Eur J Ophthalmol 2009;19:1055-1062. 
Shirakata et al.: PPV Combined with ILM Peeling to Treat Persistent Macular Edema after Anti-VEGF Treatment in Cases of Ischemic Central Retinal Vein Occlusion

9 Mandelcorn MS, Mandelcorn E, Guan K, Adatia FA: Surgical macular decompression for macular edema in retinal vein occlusion. Can J Ophthalmol 2007;42:116-122.

10 Mandelcorn MS, Nrusimhadevara RK: Internal limiting membrane peeling for decompression of macular edema in retinal vein occlusion: a report of 14 cases. Retina 2004;24:348-355.

11 DeCroos FC, Shuler RK Jr, Stinnett S, Fekrat S: Pars plana vitrectomy, internal limiting membrane peeling, and panretinal endophotocoagulation for macular edema secondary to central retinal vein occlusion. Am J Ophthalmol 2009;147:627-633.e1.

12 Radetzky S, Walter P, Fauser S, Koizumi K, Kirchhof B, Joussen AM: Visual outcome of patients with macular edema after pars plana vitrectomy and indocyanine green-assisted peeling of the internal limiting membrane. Graefes Arch Clin Exp Ophthalmol 2004;242:273-278.

-13 Baharivand N, Hariri A, Javadzadeh A, Heidari E, Sadegi K: Pars plana vitrectomy and internal limiting membrane peeling for macular edema secondary to retinal vein occlusion. Clin Ophthalmol 2011;5:10891093.

14 Yunoki T, Mitarai K, Yanagisawa S, Kato T, Ishida N, Hayashi A: Effects of vitrectomy on recurrent macular edema due to branch retinal vein occlusion after intravitreal injection of bevacizumab. J Ophthalmol 2013;2013:415974.

15 Evaluation of grid pattern photocoagulation for macular edema in central vein occlusion. The Central Vein Occlusion Study Group M Report. Ophthalmology 1995;102:1425-1433.

16 Ip MS, Scott IU, VanVeldhuisen PC, Oden NL, Blodi BA, Fisher M, Singerman LJ, Tolentino M, Chan CK, Gonzalez VH: A randomized trial comparing the efficacy and safety of intravitreal triamcinolone with observation to treat vision loss associated with macular edema secondary to central retinal vein occlusion: the Standard Care vs Corticosteroid for Retinal Vein Occlusion (SCORE) study report 5. Arch Ophthalmol 2009;127:1101-1114.

17 Brown DM, Campochiaro PA, Singh RP, Li Z, Gray S, Saroj N, Rundle AC, Rubio RG, Murahashi WY; CRUISE Investigators: Ranibizumab for macular edema following central retinal vein occlusion: six-month primary end point results of a phase III study. Ophthalmology 2010;117:1124-1133.e1.

-18 Campochiaro PA, Sophie R, Pearlman J, Brown DM, Boyer DS, Heier JS, Marcus DM, Feiner L, Patel A; RETAIN Study Group: Long-term outcomes in patients with retinal vein occlusion treated with ranibizumab: the RETAIN study. Ophthalmology 2014;121:209-219.

19 Tachi N, Hashimoto Y, Ogino N: Vitrectomy for macular edema combined with retinal vein occlusion. Doc Ophthalmol 1999;97:465-469.

20 Kado M, Jalkh AE, Yoshida A, Takahashi M, Wazen N, Trempe CL, Schepens CL: Vitreous changes and macular edema in central retinal vein occlusion. Ophthalmic Surg 1990;21:544-549.

-21 Furukawa M, Kumagai K, Ogino N, Uemura A, Larson E: Long-term visual outcomes of vitrectomy for cystoid macular edema due to nonischemic central retinal vein occlusion. Eur J Ophthalmol 2006;16:841-846.

-22 Ota M, Tsujikawa A, Kita M, Miyamoto K, Sakamoto A, Yamaike N, Kotera Y, Yoshimura N: Integrity of foveal photoreceptor layer in central retinal vein occlusion. Retina 2008;28:1502-1508.

23 Baseline and early natural history report. The Central Vein Occlusion Study. Arch Ophthalmol 1993;111: 1087-1095.

24 Natural history and clinical management of central retinal vein occlusion. The Central Vein Occlusion Study Group. Arch Ophthalmol 1997;115:486-491.

-25 Korobelnik JF, Holz FG, Roider J, Ogura Y, Simader C, Schmidt-Erfurth U, Lorenz K, Honda M, Vitti R, Berliner AJ, Hiemeyer F, Stemper B, Zeitz O, Sandbrink R; GALILEO Study Group: Intravitreal aflibercept injection for macular edema resulting from central retinal vein occlusion: one-year results of the phase 3 GALILEO study. Ophthalmology 2014;121:202-208.

-26 A randomized clinical trial of early panretinal photocoagulation for ischemic central vein occlusion. The Central Vein Occlusion Study Group N report. Ophthalmology 1995;102:1434-1444.

27 Adelman R, Parnes A, Michalewska Z, Parolini B, Boscher C, Ducournau D: Strategy for the management of diabetic macular edema: the European VitreoRetinal Society macular edema study. Biomed Res Int 2015;2015:352487.

28 Adelman RA, Parnes AJ, Bopp S, Saad Othman I, Ducournau D: Strategy for the management of macular edema in retinal vein occlusion: the European VitreoRetinal Society macular edema study. Biomed Res Int 2015;2015:870987. 
Shirakata et al.: PPV Combined with ILM Peeling to Treat Persistent Macular Edema after Anti-VEGF Treatment in Cases of Ischemic Central Retinal Vein Occlusion

Table 1. Baseline characteristics of eyes treated with PPV combined with ILM peeling for persistent ME in eyes with ischemic CRVO

Age, years

Gender, women/men

Hypertension

Diabetes mellitus

VA, $\log M A R$

CRT, $\mu \mathrm{m}$

Foveal cystoid spaces

Serous retinal detachment under the fovea

Subretinal hemorrhage under the fovea

Follow-up period, months
$67.6 \pm 11.6$

$7 / 8$

8 eyes $(53.3 \%)$

4 eyes $(26.7 \%)$

$0.88 \pm 0.57$

$566 \pm 193$

15 eyes $(100.0 \%)$

8 eyes $(53.3 \%)$

3 eyes $(20.0 \%)$

$21.2 \pm 12.9$

Table 2. Treatments before PPV combined with ILM peeling for persistent ME in eyes with ischemic CRVO

\begin{tabular}{ll}
\hline Intravitreal injections of bevacizumab & 14 eyes \\
$\quad$ Number of injections (range) & $1.9 \pm 1.2(1-5)$ \\
Intravitreal injections of ranibizumab & 2 eyes \\
$\quad$ Number of injections (range) & $0.4 \pm 1.2(2-4)$ \\
Panretinal laser photocoagulation & 9 eyes \\
Grid laser photocoagulation & 0 eye \\
Sub-Tenon injections of triamcinolone acetonide & 3 eyes \\
$\quad$ Number of injections (range) & $0.3 \pm 0.6(1-2)$ \\
$\quad$ Duration between the initiation of anti-VEGF & $6.5 \pm 4.7$ \\
$\quad$ treatment and PPV with ILM peeling, & $(1-16)$ \\
$\quad$ months (range) & \\
\hline
\end{tabular}

Table 3. Change in CRT and VA after the initiation of treatment for ME in eyes with ischemic CRVO

\begin{tabular}{lll}
\hline & CRT, $\mu \mathrm{m}$ & VA, logMAR \\
\hline Before the initiation of anti-VEGF treatment & $566 \pm 193$ & $0.88 \pm 0.57$ \\
1 month after initiation of anti-VEGF treatment & $396 \pm 159 \mathrm{a}$ & $0.85 \pm 0.46$ \\
Before PPV combined with ILM peeling & $605 \pm 181$ & $1.01 \pm 0.46$ \\
1 month after PPV combined with ILM peeling & $467 \pm 200$ & $0.95 \pm 0.48$ \\
3 months after PPV combined with ILM peeling & $446 \pm 172$ & $0.93 \pm 0.41$ \\
6 months after PPV combined with ILM peeling & $406 \pm 160^{\mathrm{b}}$ & $0.93 \pm 0.51$ \\
At final visit & $374 \pm 143^{\mathrm{a}, \mathrm{c}}$ & $1.01 \pm 0.51$ \\
\hline
\end{tabular}

${ }^{\mathrm{a}} \mathrm{p}<0.05$ (compared with the values before the initiation of anti-VEGF treatment); ${ }^{\mathrm{b}} \mathrm{p}<0.05,{ }^{\mathrm{c}} \mathrm{p}<0.01$ (compared with the values before PPV combined with ILM peeling). 
Case Reports in

Ophthalmology
Case Rep Ophthalmol 2016;7:1-8

DOI: $10.1159 / 000443322$

(c) 2016 The Author(s). Published by S. Karger AG, Basel www.karger.com/cop

Shirakata et al.: PPV Combined with ILM Peeling to Treat Persistent Macular Edema after Anti-VEGF Treatment in Cases of Ischemic Central Retinal Vein Occlusion
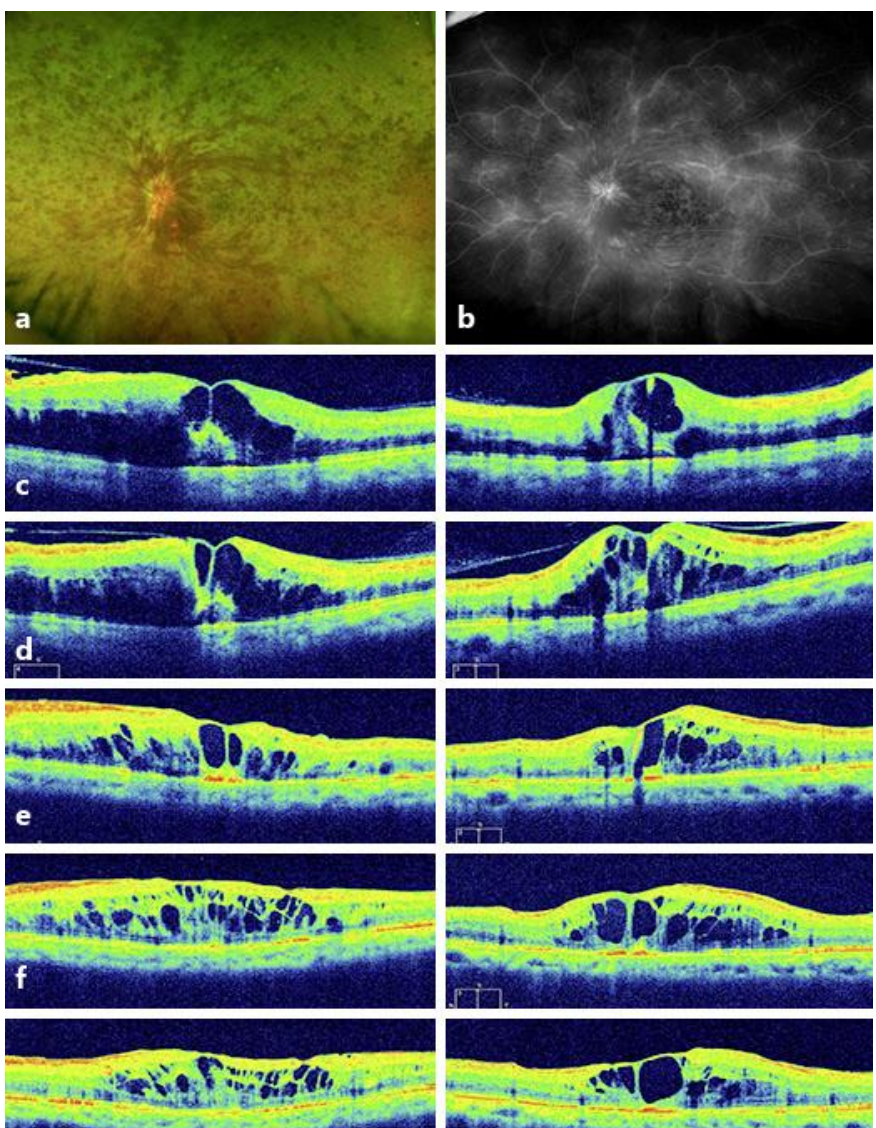

g
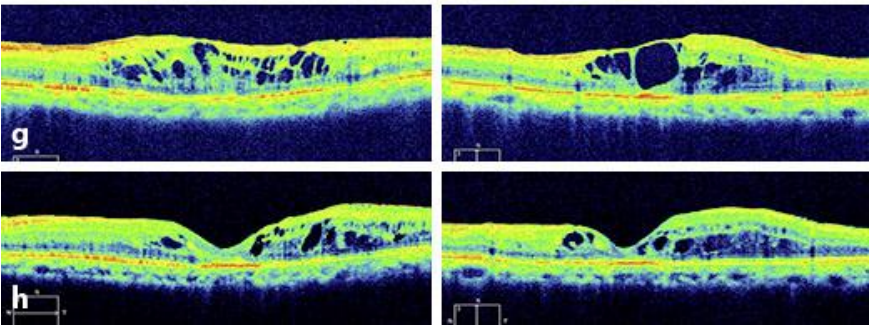

Fig. 1. A 64-year-old man had decreased VA in the left eye (0.3 OS) due to ME caused by ischemic CRVO. a Fundus photograph at the initial visit showing extensive retinal hemorrhage. b Fluorescein angiogram showing a large non-perfused area. c-h Horizontal (left) and vertical (right) sectional images centered on the fovea were obtained by OCT. c OCT section at initial visit showing ME (CRT $=402 \mu \mathrm{m}$ ). $\mathbf{d}$ No reduction in ME was seen after an intravitreal injection of bevacizumab (CRT $=403 \mu \mathrm{m}$ ). There was also no improvement in VA (0.2 OS). Three months after the initial visit, the left eye was treated with PPV with ILM peeling. During surgery, laser photocoagulation was performed on the non-perfused area. $\mathbf{e}-\mathbf{h}$ After surgery, ME decreased gradually, with no visual improvement. e One month after surgery (CRT $=484 \mu \mathrm{m}$, $0.1 \mathrm{OS}$ ). $\mathbf{f}$ Three months after surgery (CRT $=567 \mu \mathrm{m}, 0.2 \mathrm{OS}$ ). $\mathbf{g}$ Six months after surgery (CRT $=456 \mu \mathrm{m}$ 0.2 OS). $\mathbf{h}$ Twelve months after surgery (CRT $=279 \mu \mathrm{m}, 0.15$ OS). 